Société d'histoire de la révolution de 1848 et des

révolutions du XIXe siècle

$47 \mid 2013$

Quel est l'avenir du XIX ${ }^{\mathrm{e}}$ siècle ?

\title{
Le loup de Dickens
}

\section{Evelyne Loew}

\section{OpenEdition \\ Journals}

Édition électronique

URL : http://journals.openedition.org/rh19/4547

DOI : $10.4000 /$ rh 19.4547

ISSN : $1777-5329$

Éditeur

La Société de 1848

Édition imprimée

Date de publication : 31 décembre 2013

Pagination : 51-57

ISSN : 1265-1354

Référence électronique

Evelyne Loew, "Le loup de Dickens », Revue d'histoire du XIXe siècle [En ligne], 47 | 2013, mis en ligne le 31 décembre 2016, consulté le 30 avril 2019. URL : http://journals.openedition.org/rh19/4547 ; DOI : $10.4000 /$ rh19.4547 


\title{
EVELYNE LOEW
}

\section{Le loup de Dickens}

\begin{abstract}
«Économistes utilitaristes, maîtres d'école décharnés, délégués aux Faits, incroyants distingués de force credo éculés, vous aurez toujours des pauvres avec vous. Tandis qu'il en est temps encore, cultivez en eux toutes les grâces de l'imagination et du sentiment pour orner leurs vies si dénuées d'ornements, sinon, au jour de votre triomphe, lorsque le goût du merveilleux aura été à tout jamais chassé de leurs âmes, et qu'ils se retrouveront face à face avec leur existence dénudée, la Réalité se changera en loup et vous dévorera.» (Charles Dickens, Temps difficiles [Hard Times], 1854, traduction française Andhrée Vaillant, Gallimard, Folio, 1985)
\end{abstract}

\section{Morale individuelle et Collective}

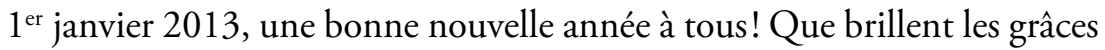
de l'imagination et du sentiment! En ce temps de confusion et de profusion, de désarroi, de bouleversements de la vie quotidienne, de changement de la conception même du travail - la troisième révolution industrielle est passée par là -, en ce temps qui offre chaque jour, via le journal ou Internet, une récolte d'informations promettant le pire ou le meilleur - nul ne saurait le dire - temps de grandes espérances confuses, nous revenons aux fondamentaux. Comme Charles Dickens, qui vécut en personne l'instauration d'une main de fer de la nouvelle ère de la grande industrie capitaliste, à un âge où l'on est sensible et vulnérable - douze ans - en collant des étiquettes sur des bouteilles de cirage dans une usine sordide de la banlieue de Londres tandis que sa famille était en prison pour dettes, comme Dickens donc nous sentons vivement que nous sommes au début d'un nouveau cycle. Lequel? Au $\mathrm{XIX}^{\mathrm{e}}$ siècle, la société industrielle devient un fait, au XXI ${ }^{\mathrm{e}}$ siècle la métamorphose en profondeur de la société industrielle est devenue un fait. Comme Dickens, nous sommes en quête de la morale individuelle et collective qui fasse barrage aux égoïsmes avides et aveugles. En quête du merveilleux, non marchand, non religieux, non dogmatique, qui enchantera nos vies. 
Au mitan du XIXe siècle la lutte syndicale et les idéologies qui vont croître et embellir au siècle suivant n'en sont qu'à leurs balbutiements. C'est dans les ressources intérieures, dans l'énergie d'une résistance personnelle et la force des valeurs de compassion, de partage, de droiture, que Dickens trouve toutes les raisons d'espérer. Ces mêmes valeurs sont défendues, à la même époque (1840) en France, par George Sand, par exemple dans son roman Le Compagnon du Tour de France, une ouvre peu connue basée sur une enquête passionnante.

Après les séismes du XXe siècle et les idéologies centrifugeuses, nous voici, encore et toujours, revenus aux bonnes volontés, aux valeurs morales, à l'association des hommes et des femmes de bonne volonté dans des actions immédiates, modestes peut-être, mais aux résultats tangibles.

\section{Sur LES PLANCheS}

J'ai écrit trois pièces de théâtre mettant en scène le mitan du XIX ${ }^{\mathrm{e}}$ siècle. Marigold, d'après Charles Dickens, Le Banquet des utopies, sur les courants politiques avant 1848, Le Procès d'un grand rêve, sur le procès à grand spectacle des insurgés du 15 mai 1848 qui eut lieu en 1849 à Bourges, au Palais Jacques-Cour aménagé pour l'occasion en Haute Cour de Justice. La pièce, mise en scène par George Buisson, s'est jouée dans le lieu même du procès.

Ces trois pièces ont été jouées ou lues de nombreuses fois, suivies la plupart du temps de débats. Lors de ces débats, la prise en compte immédiate, actuelle, des questionnements était frappante. Un foisonnement de questions, de réflexions, des discussions passionnées, les interventions de militants associatifs, politiques, de citoyens curieux de tout comme il y en a tant, un grand appétit pour ce moment de l'histoire, de nombreuses demandes de bibliographie.

Le Banquet des utopies, lui, après avoir été créé par une merveilleuse équipe de comédiens amateurs à la Maison de George Sand, a été repris par les élèves d'une classe entière d'un collège de Saint-Benoît-du-Sault, mis en scène par Baptiste Kubich. Ces jeunes se sont emparés avec un plaisir et un intérêt incroyables de cette histoire qui est la leur. Une histoire pourtant fort complexe dont ils ne connaissaient quasiment rien. C'était beau de voir la fierté qu'ils avaient à la faire découvrir aux autres élèves, à leurs parents, au public de la ville.

Bien sûr, cela tient à la forme théâtrale elle-même, à l'immédiateté de la présence des comédiens, au charisme de la mise en scène, à la projection des préoccupations de l'observateur, en l'occurrence moi-même. Le théâtre est un art qui a la vertu d'agir au présent. Mais je suis persuadée que cela tient aussi à la proximité des préoccupations d'une époque qui nous a nourris profondément et qui a été écrasée dans la mémoire collective par les deux 
montagnes que sont la Révolution française, avant, et la lutte des classes version marxiste, après.

Nous retrouvons aujourd'hui seulement, hors des orthodoxies politiques, les interrogations existentielles sur des moyens d'action qui soient efficaces face à l'égoïsme autoproclamé d'une organisation sociale cynique. Nous retrouvons la force de l'humour aussi, comme arme par défaut contre les turpitudes des puissants. Le succès sur les plateaux des pièces de Feydeau, dénonciateur des tripatouillages et absurdités de son époque, revisitées par de décapantes et jubilatoires mises en scène, témoignent du même phénomène. En tout état de cause, les concordances entre milieu du XIX ${ }^{\mathrm{e}}$ et début $\mathrm{du} \mathrm{XXI}^{\mathrm{e}}$ siècle contribuent à donner des forces et de l'optimisme. Comme si la réflexion, revenant pensivement avant 1848 - date fatidique de la faillite des espoirs d'un socialisme humanitaire et réformiste, faillite dont Marx et Engels ont su tirer des conséquences lourdes de réorientations pour les générations à venir - pouvait se permettre à nouveau de rêver à un avenir au croisement d'utopies non-violentes.

\section{Attention aUX SCÉnarios De L'AVEnir!}

Ces utopies de la première moitié du XIX ${ }^{\mathrm{e}}$ siècle? Elles ont été réalisées partiellement. Elles ont parfois dépassé leurs objectifs. Les rêves de nos arrière-grands-pères et arrière-grands-mères ont pris forme, des formes parfois monstrueuses, parfois heureuses. N'en est-il pas toujours ainsi? Les rêves d'une génération se réalisent à un siècle de distance. Prêtons donc plus d'attention à nos rêves! Essayons d'évaluer sincèrement leurs charges positives, négatives, fructueuses, destructrices.

Rien de plus stimulant que de lire les utopistes du début du XIX ${ }^{e}$ siècle. Une époque riche en systèmes se réclamant tous de la science triomphante et du progrès. Voyage en Icarie (1845), d'Etienne Cabet, est un gros volume qui propose un voyage dans un pays mirifique, vitrine d'une communauté idéale. Dix-neuf chapitres détaillés qui balaient tous les aspects de la vie. Un livre qui eut un grand succès, une sorte de Bible lue à haute voix par les travailleurs éclairés. Une Bible qui aujourd'hui consterne. Nous y découvrons une sorte de manuel anticipé des khmers rouges ou de la révolution culturelle maoïste et autres dérives totalitaires. En échange d'une sécurité sociale - pas de chômage, logement attribué à tous, hôpital gratuit, absence d'argent, nivellement des conditions - Cabet instaure, en toute bonne conscience, le contrôle bureaucratique absolu, social et intime. Tout est réglementé. Le travailleur doit promettre de se soumettre dans la joie aux réquisitions de la collectivité. Prêter serment. Sa vie est totalement transparente. Nous voilà loin des principes des Lumières, des Droits de l'homme, loin des débats de la Convention. En Icarie, tous travaillent en chantant (c'est obligatoire), au 
son de la cloche ou de la trompette, et mangent en commun des menus «hygiéniques» dans d'immenses cantines. Interdiction de rester célibataire, accouplement obligatoire. Des escouades d'artistes célèbrent Icarie, ils ont rejeté la mollesse et la sensualité des arts «décadents».

La seule chose qui reste aujourd'hui pittoresque et délicieuse en Icarie, outre sa naïveté, est l'invention d'une langue internationale, une sorte d'espéranto, une langue rapide et pratique, basée sur les racines de toutes les langues existantes, sans grammaire ni orthographe. Ainsi les travailleurs de tous les pays qui rejoignent Icarie peuvent se comprendre en un clin d'œil. Quoique peut-être la simplification extrême de cette langue puisse être un tout petit peu inquiétante, non?

Les témoignages sur la condition ouvrière au début de l'ère industrielle, comme les palpitants Mémoires et aventures d'un prolétaire à travers la révolution du très intelligent Norbert Truquin, ou les enquêtes de Flora Tristan, nous permettent de nous identifier aux ouvriers de l'époque et de comprendre aisément que l'idée de bénéficier d'un logement gratuit sain et aéré avec eau courante et tout-à-l'égout, l'idée de manger deux fois par jour, l'idée de ne plus pâtir du chômage, aient pu exalter et aveugler les canuts lyonnais ou les ouvriers des filatures de Rouen terrés dans des taudis infects, mangeant dans les meilleurs jours de la retaille de viande. Mais cela n'empêche pas de frémir en voyant se dévoiler ainsi, dès 1845 , le pire du pire derrière une organisation aussi rigide et aussi contraire à la diversité de la vie. Oui, l'utopie icarienne a été réalisée au $\mathrm{XX}^{\mathrm{e}}$ siècle, avec pertes et fracas.

Une autre utopie réalisée au XXe siècle, à l'autre extrémité du spectre, c'est l'utopie fouriériste. Dans un supermarché, dans un hypermarché, je vois surgir le spectre ravi de Charles Fourier, lui qui a tant rêvé de centaines de variétés de crèmes et de laitages en se léchant les babines par défaut au-dessus de son manuscrit, lui qui a célébré la gourmandise, lui qui a prôné comme base de l'éducation la cuisine et l'opéra. Je le vois, notre Fourier national, dansant d'allégresse au milieu des rayons. Dans les immenses cavernes d'Ali Baba d'aujourd'hui, remplies de denrées venues des quatre coins du monde, dans ces palais des merveilles qu'aucun roi du passé, aussi riche soit-il, n’a égalés, et qui ne nous étonnent plus, Charles Fourier exulterait! Lui qui disait qu'il fallait au moins cent variétés de soupes pour satisfaire le public. Il avait prédit la fonte des glaces et l'ouverture d'une nouvelle voie de navigation par le Pôle Nord. Nous n'en sommes pas encore à son émoustillante transformation de l'eau de mer en limonade à volonté, ni à ses couloirs aériens autoportants chauffés, mais cela ne saurait tarder. Quant au bouleversement des mœurs, il en fut le précurseur. Mai 68 en France lui aura rendu justice en reprenant nombre de ses aphorismes. 
Sous les PAVÉS AU XIX ${ }^{\mathrm{e}}$, LA Plage DU XXI ${ }^{\mathrm{e}}$ ?

L'égalité des femmes et des hommes - on en était bien loin au début du $\mathrm{XIX}^{\mathrm{e}}$ siècle -, la liberté des mœurs (pour Fourier rien n'est interdit à partir de l'âge de dix-huit ans, âge fixé par lui comme l'âge adulte), la reconnaissance de toutes les tendances et passions - les «comaniens» chez Fourier sont les personnes qui ont les mêmes passions ou les mêmes manies, dans quelque domaine que ce soit, et qui se retrouvent, s'associent pour en jouir, en toute liberté du moment qu'ils ne nuisent pas à autrui -, ces grands thèmes d'unité et d'harmonie universelles fouriéristes se sont installés au cour du monde occidental via les médias, le Web, et la culture de masse. Avec une infantilisation certaine, déjà bien présente chez Fourier. Par contre, la croyance fouriériste, optimiste et résolue, que toute tendance perverse, négative, malveillante ou destructrice, s'éteindrait d'elle-même dans une société d'abondance et d'hédonisme, ne s'est pas du tout, mais alors pas du tout réalisée!

Les courants saint-simoniens nécessiteraient un article entier. Eux aussi reconnaîtraient leurs petits aujourd'hui et crieraient victoire. D'ailleurs, ayant bifurqué pour la plupart sur la voie des réalisations concrètes (les canaux, les chemins de fer, les grands travaux de toutes sortes) ils ont pu voir de leur vivant la réalisation de leurs rêves.

Quant à Proudhon, il fut bien le premier à prôner le microcrédit. Nos économistes, s'ils puisent allègrement dans ses propositions, le citent trop peu. Il fut le défenseur acharné de la décentralisation, une idée neuve promise à un bel avenir, et de la relocalisation. Sans parler de l'autogestion. Pour lui une véritable démocratie ne pouvait se baser que sur des communautés et des systèmes de contrôles restant à portée de main (à portée de clic de souris aujourd'hui?).

Il y a aussi la cohorte des visionnaires anti-technique, anti-progrès, qui ne manqueraient pas de rappeler leurs prophéties apocalyptiques, faisant fi des énormes progrès de l'hygiène, de la médecine, de la sécurité et de la dignité de la vie.

Le pire et le meilleur. Victor Hugo, dans le dernier poème de La Légende des siècles, "Plein Ciel", évoque un $\mathrm{XX}^{\mathrm{e}}$ siècle où l'homme, conquérant du ciel, voyage à son gré et unifie le globe. Sans supposer un instant que de ces prouesses d'invention, de cette exaltation pouvait naître le malheur absolu des bombardements massifs et d'Hiroshima.

\section{Nos CONTEMPORAINS DU XIX ${ }^{\mathrm{e}}$ SIÈCLE}

Avant d'écrire et de travailler pour et dans le théâtre, il se trouve que j'étais ouvrière, entre 1969 et 1977, dans une entreprise de la métallurgie de la vieille «banlieue rouge» au Nord de Paris. À ce moment-là, je m’en 
souviens très bien, j'avais souvent l'impression de vivre les derniers jours du $\mathrm{XIX}^{\mathrm{e}}$ siècle. Le travail au rendement, les accidents, une vie toute dessinée, la culture et la fierté ouvrières, républicaines, les liens de solidarité et d'amitié inébranlables forgés à l'atelier. Un monde de luttes et de conquêtes sociales à l'orée duquel se trouvent aujourd'hui les ouvriers, les mineurs, les travailleurs, de Chine, d'Inde ou d'Afrique. Avec à l'ordre du jour l'éradication du travail des enfants, la diminution des horaires de travail, la sécurité sur les machines, la protection sociale, la lutte contre l'esclavage et l'emprise de l'usine. Les grandes conquêtes législatives de la fin du XIX ${ }^{e}$ siècle (les lois sur le travail des enfants et l'instauration progressive du Code du travail en France), loin d'être acquises dans le monde entier, restent d'une actualité brûlante. En France même, avec la précarisation généralisée, le chômage, le travail à temps partiel, les jeunes stagiaires non payés, les contrats de mission, la période d'activité réduite à quelques années, grosso modo entre 30 ans et 50 ans (!), la mise en concurrence des travailleurs, un grand cycle industriel est terminé, certes, mais ne croirait-on pas revenus les temps terribles du marchandage?

Le XIX ${ }^{e}$ siècle m'a semblé également contemporain quand j'ai interviewé, pour le théâtre cette fois, de nombreuses personnes âgées à Châtenay-Malabry, en banlieue parisienne, en 1979-1980-81, dans le cadre d'un projet intitulé Une ville se raconte, financé par le Fonds d'intervention culturelle. Des centaines d'enregistrements dont naîtront de superbes pièces de théâtre, de formes très différentes, représentées devant les habitants de Châtenay puis à travers toute la France, mises en scène par Jean-Claude Penchenat, jouées par les comédiens professionnels du théâtre du Campagnol et par de nombreux comédiens amateurs de Châtenay.

Les personnes interviewées - ordinaires et extraordinaires, comme nous le sommes tous - étaient nées à la fin du XIX ${ }^{e}$ siècle. L'une n'avait pas oublié sa petite robe rouge, confectionnée par sa mère, portée pour l'enterrement de Louise Michel en 1905, l'autre le remplissage des lampes à pétrole, la pesée du pain et les plats à base d'épluchures de pommes de terre. Je réalise maintenant seulement combien cette transmission en direct de l'histoire a été merveilleuse, vivante, drôle, inattendue, profondément marquante. Une transmission orale chargée d'émotions, qui, la plupart du temps, ne passe plus par les familles, mais qui peut continuer via le théâtre, l'école, les bibliothèques, les rencontres organisées de manière à faire parler - et surtout à faire écouter! - les personnes âgées.

\section{UN PAYS OÙ IL FAUT VIVRE POUR LE CONNAÎTRE}

Le XIX e ? C'est un continent où vivent les historiens qui n'ont pas fini de faire des découvertes et d'en rendre compte, région par région, secteur par secteur, pour notre bonheur, notre instruction et notre curiosité. N'étant pas 
historienne, je ne veux pas ressembler, dans cet article, au touriste qui, ayant visité un petit quartier de Bombay, aurait la prétention d'émettre un avis sur l'Inde! Consciente de l'étroitesse de mon angle de vision, je réclame la bienveillance du lecteur. J'ai voulu relater, tout simplement, quelques échos du XIX ${ }^{\mathrm{e}}$ siècle dans ma vie.

En conclusion, de ces batailles initiées au XIX ${ }^{\mathrm{e}}$ siècle, il me semble qu'une a déjà été gagnée, celle des valeurs, celle de l'esprit. Grâce, entre autres, à des œuvres de fiction qui ont nourri notre imaginaire et l'imaginaire collectif du monde entier, les grands romanciers du XIX ${ }^{\mathrm{e}}$ siècle sont devenus pour nous l'équivalent des penseurs de l'Antiquité pour les esprits de la Renaissance. Une ressource, une base, une force. Le consensus n'est pas encore là, mais leur influence s'est élargie à l'univers en faisant avancer, sinon dans les faits du moins dans les aspirations, les idées de diversité, de tolérance et de justice sociale. C'est déjà beaucoup.

Evelyne Loew est dramatuge; parmi ses pièces, certaines mettent en scène des épisodes du XIXe siècle: Gorki-Tchekhov, 1900, Actes Sud-Papiers (1997), Le Banquet des utopies, Les mille univers, Éditions du patrimoine, Centre des Monuments Nationaux — Maison de George Sand (2008), Le Procès d'un grand rêve, Éditions de Champtin /Éditions du patrimoine, Centre des Monuments Nationaux — Palais Jacques-Cour (2007), Marigold (inédit) 